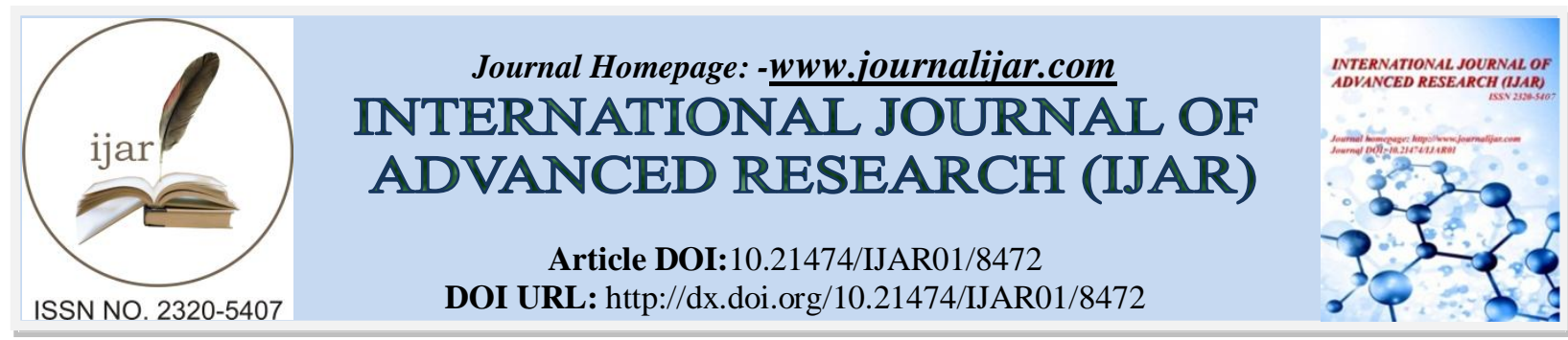

RESEARCH ARTICLE

\title{
STUDY OF URINARY TRACT INFECTIONS IN TERTIARY CARE HOSPITAL EMPHASIZING ON BACTERIOLOGICAL PROFILE WITH ANTIBIOGRAM.
}

Swathi Kuna, P. Ratna Kumari and R.Lakshmi Kumari.

Department of Microbiology ,Siddhartha Medical College,Vijayawada-520008.

\section{Manuscript Info}

Manuscript History

Received: 02 December 2018

Final Accepted: 04 January 2019

Published: February 2019

Key words:-

Infection, Escherichia coli, empirical antibiotics.

\begin{abstract}
The present study was carried out to analyse the culture reports 470 urine samples collected from symptomatic patients between 18 to 80 years from department of medicine, surgery and gynaecology from May 2017 to 0ctober 2017. All the samples collected during the above period were subjected for culture sensitivity as per standard methods. The most common infection encountered in clinical practice is urinary tract infection. Urinary tract infection can be caused by different microorganisms including bacteria, parasites, fungi and viruses which are the major causative organisms. Among these, bacteria account for more than $95 \%$ of Urinary tract infection cases. Escherichia coli is the commonest urinary pathogen accounting for over $80 \%$ of community acquired infection. In this study the most common pathogenic organism isolated was Escherichia coli 44(55.69\%) followed by Staphylococcus aureus 20(25.31\%) and Klebsiella species 11(13.92\%) in both males and females. Escherichia coli were sensitive to nitrofurantoin, amikacin and piperacillin tazobactam in 35 (79.4\%), 25(56.81\%) and $18(40.95 \%)$ respectively. Similarly $16(80 \%)$ of Staphylococcal isolates were sensitive to nitrofurantoin $11(55 \%)$ to amikacin and $5(25 \%)$ to piperacillin tazobactam. Klebsiella isolates also showed highest sensitivity to nitrofurantoin and piperacillin tazobactam $7(63 \%)$ followed by ceftriaxone 6 ( $54 \%$.) This study will help clinicians to choose the right empirical antibiotics, and they should take into account the local sensitivity and resistance patterns while prescribing empirical oral or intravenous antibiotics.
\end{abstract}

Copy Right, IJAR, 2019,. All rights reserved.

\section{Introduction:-}

Urinary tract infections (UTIs) are one of the most common bacterial infections in humans, both in the community and hospital setting, and it is one of the leading hospital acquired infections. UTI can be caused by different microorganisms including bacteria, fungi, parasites, and viruses, of these bacteria account for more than $95 \%$ of cases. Normally, natural commensals protect us from infections. In case of UTI, Lactobacillus species is the commensal. But these natural commensals get destroyed by antibiotics. UTI can be symptomatic or asymptomatic. UTI are classified into complicated and uncomplicated. Uncomplicated UTI is said to be present if there are no functional and anatomical anomalies in the urinary tract or no renal functional impairment. A lower UTI (Acute Uncomplicated Cystitis) is taken to be present when symptoms are restricted to the lower urinary tract, (dysuria, 
urgency or pain) above the symphysis. An upper UTI (Acute Uncomplicated Pyelonephritis) is present when symptoms also include, flank pain, and/or fever $\left(>38^{\circ} \mathrm{C}\right)$. Complicated UTI include infection in persons with anatomical abnormalities, obstruction, hydronephrosis, renal tract calculi, or colovesical fistula, in patients with an immune compromised state, atypical organisms causing UTI, in pregnancy and UTI are occurring after instrumentation, surgeries and radiotherapy. Complicated one's may not be controlled with single antibiotic but may need multi drug therapy which may be usually IV antibiotics. The quantitative criteria of at least $10^{8}$ colony forming units $(\mathrm{cfu}) / \mathrm{L}$ (at least $10^{5} \mathrm{cfu} / \mathrm{mL}$ ) is generally appropriate for the microbiological identification. Distribution of uropathogens and their susceptibility to antibiotics is variable regionally, so it becomes necessary to have knowledge of distribution of uropathogens and their susceptibility to antibiotics in a particular setting. Antibiotic resistance in the treatment of UTI and other bacterial infections constitute a major public health problem especially in the developing countries as irrational and indiscriminate use of antibiotics is common. In view of these and attendant tendency for changes in bacteriological profile, it is worthwhile that the degree of susceptibility and resistance of these uropathogens to various antibiotics be known to clinicians for effective treatment of infections they cause and to avoid antibiotics misuse.

\section{Methods:-}

The present study was conducted in the Department of Microbiology, Government General Hospital, Vijayawada. Urine samples collected from symptomatic patients between 18 to 80 years from different departments of hospital were analysed for culture and sensitivity between May 2017 to Oct 2017. Urine specimens received in the department of microbiology were cultured on nutrient agar and Macconkey agar for 18 to 24 hours at $37^{0} \mathrm{c}$. Then colonies were counted and colonies with more than $10^{5} \mathrm{CFU} / \mathrm{ml}$ were considered as culture positive .The positive samples were processed using biochemical tests and identification of micro organisms were done according to standard procedure. The standard disc diffusion method (kirby bauer's disc diffusion) on Muller Hinton agar was utilized for all the isolates to assess the antibiotic resistance using antibiotic discs according to recent CLSI guidelines 2017.

Figure 1:-Total number of samples $(\mathrm{n}=470)$

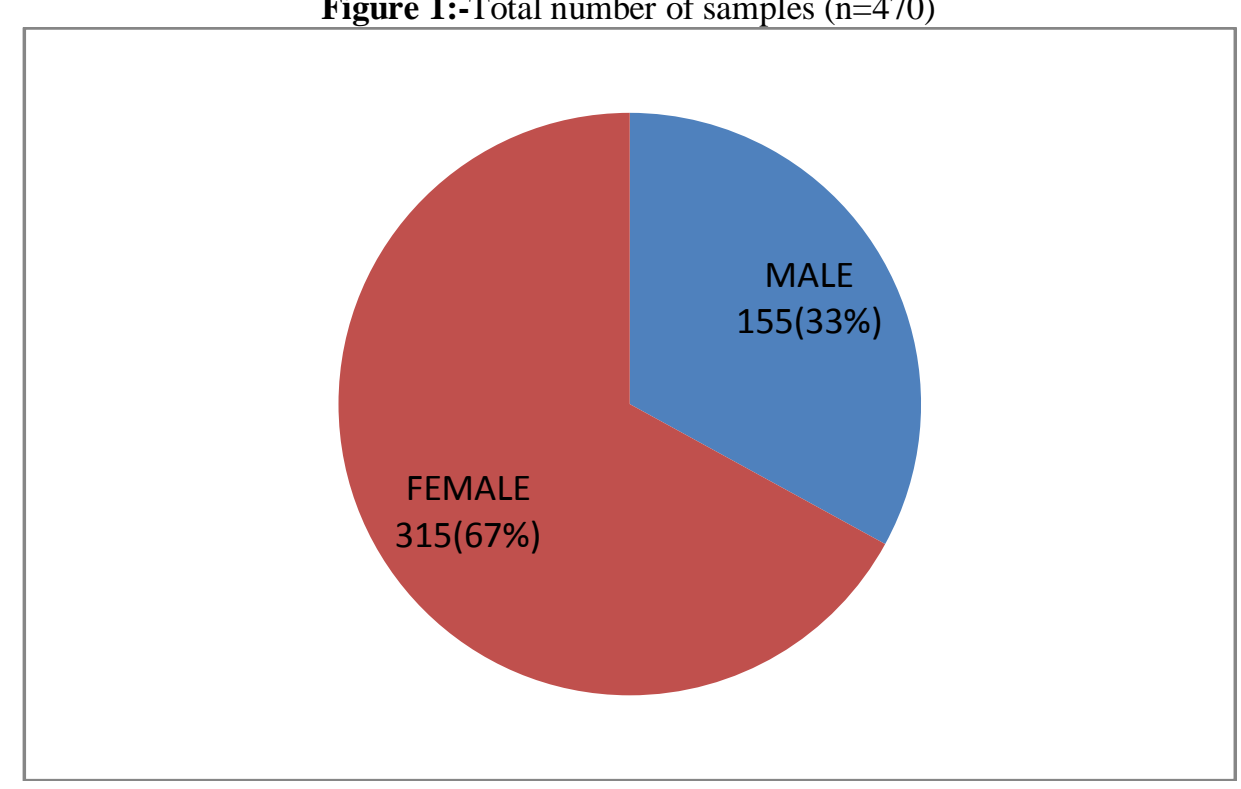


Figure 2:-Age and gender wise distribution Male $(n=155)$ Female $(n=315)$

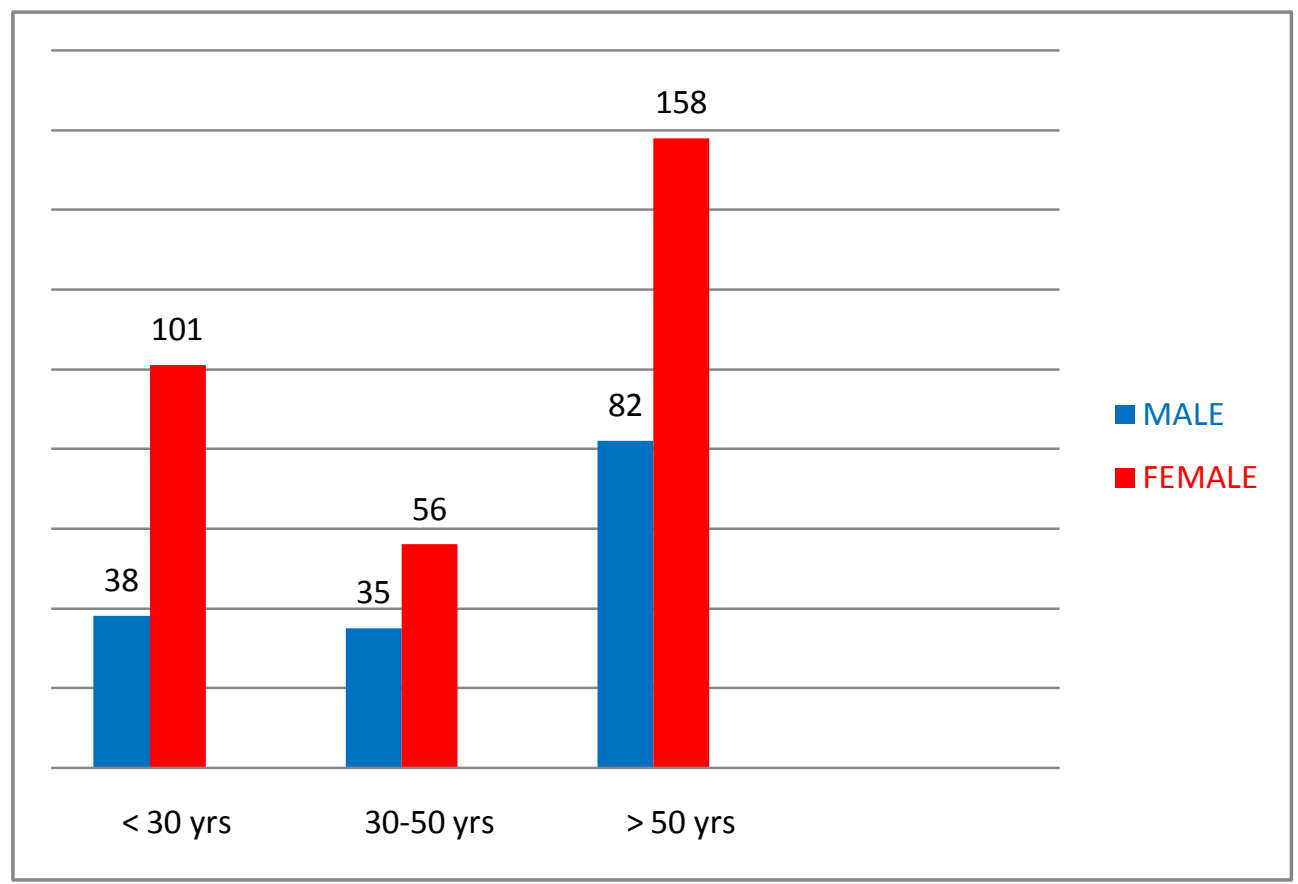

Figure 3:-Organisms isolated $(\mathrm{n}=106)$

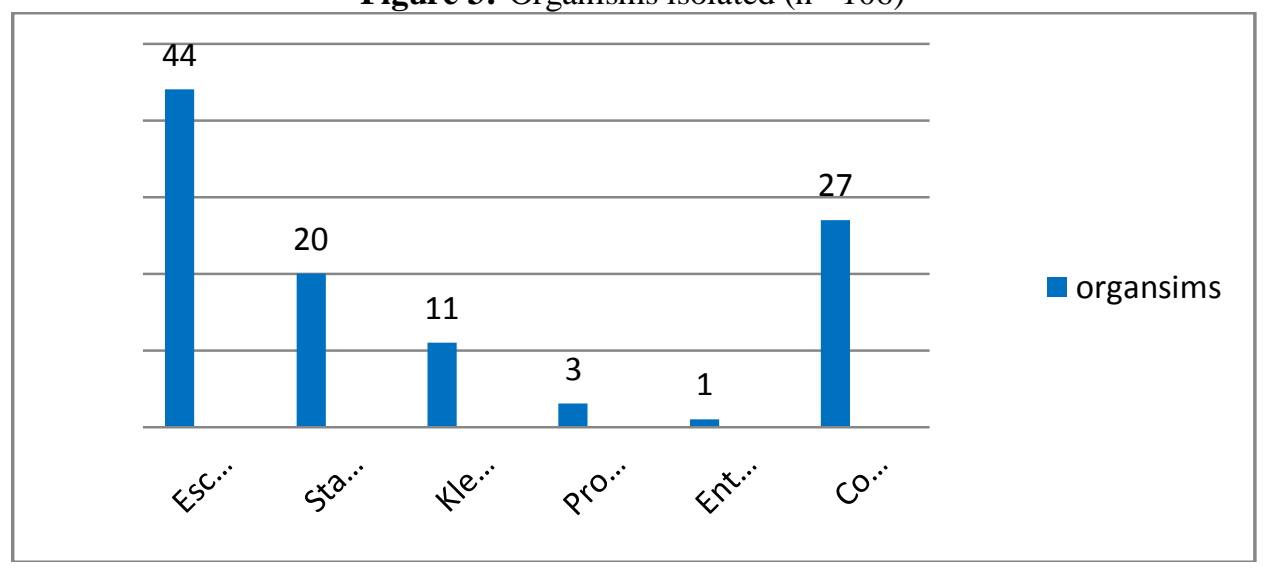

Table 1:-Antibiotic susceptibility pattern of isolates

\begin{tabular}{|l|l|l|l|l|l|}
\hline & E.coli $(44)$ & $\begin{array}{l}\text { Staphylococcus } \\
(20)\end{array}$ & Klebsiella (11) & Proteus (3) & Enterococcus(1) \\
\hline Nitrofurantoin & $35(79.54 \%)$ & $16(80.00 \%)$ & $7(63.00 \%)$ & $1(33.33 \%)$ & $1(100.00 \%)$ \\
\hline Amikacin & $25(56.81 \%)$ & $11(55.00 \%)$ & $4(36.36 \%)$ & $2(66.66 \%)$ & $1(100.00 \%)$ \\
\hline Gentamycin & $20(45.45 \%)$ & $6(30.00 \%)$ & $3(27.27 \%)$ & - & $1(100.00 \%)$ \\
\hline $\begin{array}{l}\text { Piperacillin } \\
\text { tazobactam }\end{array}$ & $18(40.90 \%)$ & $5(25.00 \%)$ & $7(63.00 \%)$ & $3(100.00 \%)$ & $1(100.00 \%)$ \\
\hline Norfloxacin & $13(29.54 \%)$ & $3(15.00 \%)$ & $4(36.36 \%)$ & $3(100.00 \%)$ & $1(100.00 \%)$ \\
\hline Cotrimoxazole & $11(25.00 \%)$ & $4(20.00 \%)$ & $3(27.27 \%)$ & - & - \\
\hline Ciprofloxacin & $818.18 \%)$ & $4(20.00 \%)$ & - & $1(33.33 \%)$ & - \\
\hline $\begin{array}{l}\text { Cefaparazone } \\
\text { sulbactam }\end{array}$ & $715.90 \%)$ & - & $4(36.36 \%)$ & $1(33.33 \%)$ & - \\
\hline Ceftriaxone & $5(11.36 \%)$ & $5(25.00 \%)$ & $6(54.54 \%)$ & $2(66.66 \%)$ & - \\
\hline
\end{tabular}




\begin{tabular}{|l|l|l|l|l|l|}
\hline Cefotaxim & $5(11.36 \%)$ & $1(5.00 \%)$ & $2(18.18 \%)$ & - & - \\
\hline Imepenam & $3(6.81 \%)$ & $1(5.00 \%)$ & $2(18.18 \%)$ & $1(33.33 \%)$ & - \\
\hline Ceftazidime & $2(4.54 \%)$ & - & $1(9.09 \%)$ & - & - \\
\hline Cefepime & $1(2.27 \%)$ & - & $1(9.09 \%)$ & - & - \\
\hline Linezolid & - & $5(25.00 \%)$ & - & $1(33.33 \%)$ & - \\
\hline Levofloxacin & - & $3(15.00 \%)$ & - & - & $1(100.00 \%)$ \\
\hline Vancomycin & - & $5(25.00 \%)$ & - & $1(33.33 \%)$ & - \\
\hline Ampicillin & - & $1(5.00 \%)$ & $1(9.09 \%)$ & - & - \\
\hline Doxycillin & - & $2(10.00 \%)$ & - & - & - \\
\hline
\end{tabular}

\section{Results:-}

Total 470 samples were collected. Among 470 samples from patients, males were $155(33 \%)$ and females were $315(67.21 \%$ ) (figure1). Females outnumbered males in all age groups (figure 2). Out Of 470, 106(22.55\%) samples were culture positive. Out of which 79(74.52\%) were pathogenic organisms, 27(25.47\%) were commensals. The most common pathogenic organism isolated was Escherichia coli 44(55.69\%) followed by Staphylococcus aureus $20(25.31 \%)$ and Klebsiella species 11(13.92\%) in both males and females (figure 2, and 3). Escherichia coli was sensitive to nitrofurantoin, amikacin and piperacillin tazobactam in 35(79.4\%), 25(56.81\%) and 18(40.95\%) respectively. Similarly $16(80 \%)$ of staphylococcal isolates were sensitive to nitrofurantoin $11(55 \%)$ to amikacin and $5(25 \%)$ to piperacillin tazobactam. Klebsiella isolates also showed highest sensitivity to nitrofurantoin and piperacillin tazobactam 7(63\%) followed by ceftriaxone 6( 54\%.) (Table 1)

\section{Discussion:-}

The present study was done to find out the sensitivity pattern of bacteria which commonly cause UTI with an aim to provide clinicians with a choice of proper empirical antibiotics. Number of studies were done all over the world on the antibiogram of UTI with different percent of culture positivity, ranging from as low as $6 \%$ to more than $80 \%$. Culture positive rate in our study is $(22.55 \%)$. Similar low culture positivity was obtained by Amarjit singh et al., where (27.10\%) of samples were culture positive. In our study female to male ratio was 2.03: 1, similar to other studies which showed female preponderance. In our study, among pathogenic organisms isolated, Escherichia coli was the commonest organism in both males and females (55.69\%). Study done by Monika yadav et al, showed that $(61 \%)$ of their isolates were Escherichia coli and another study done in North India showed (68.7\%) of their isolates were Escherichia coli. In our study (79.4\%) of Escherichia coli isolates, were sensitive to nitrofurantoin, and $(78 \%)$ Escherichia coli isolates in study done by Monika yadav et al were sensitive to nitrofurantoin. However study done by Swati talari et al, showed (100\%) of Escherichia coli were sensitive to Nitrofurantoin. In our study (56.81\%) of Escherichia coli , were sensitive to amikacin when compared to(46\%) of Escherichia coli isolated in study by Avinash et al. In both our studies $40 \%$ of Escherichia coli isolates were sensitive to Piperacillin tazobactum. However, study done by Swati et al ., showed (100\%) of Escherichia coli isolated were sensitive to amikacin and piperacillin tazobactam. In our study, all the organisms were resistant to ampicillin and amoxicillin sulbactam group of antibiotics. Similar high resistance to amoxicillin (> 85\%) was found by Mulugeta Kibret et al.,. However, a different study done by Mohmoud et al., showed that Escherichia coli, Klebsiella and staphylococcal species were sensitive to amoxicillin in more than (70\%) cases. In another study by Agnus N oli, levofloxacin was the most suitable empirical antibiotic with more than (75\%) sensitivity against most bacterial isolates. In our study, only Enterococcus species were sensitive to levofloxacin, hence cannot be recommended for empirical therapy. Though Escherichia coli, Klebsiella species and staphylococcal species were the common pathogens isolated worldwide, sensitivity patterns were different in different studies. It is important to know the local sensitivity pattern while treating UTI to start empirical antibiotics and to prevent unnecessary and improper antibiotic usage. This study will help clinicians to choose the right empirical antibiotics, awaiting culture reports. Clinicians should take into account the local sensitivity and resistance patterns while prescribing empirical oral or intravenous antibiotics for urinary tract infections.

\section{Acknowledgement:-}

I am thank full to all the doctors, technical staff of Microbiology Department, Government General Hospital, Siddhartha Medical College, Vijayawada who cooperated us in carrying out this research work. 


\section{References:-}

1. Akram M, Shahid M and Khan AU (2007). Etiology and antibiotic resistance patterns of community acquired urinary tract infections in J N M C Hospital Aligarh, India. Annals of Clinical Microbiology and Antimicrobials 64

2. Amarjit singh vij, Shashi chopra and Jigyasa sehgal. Two years retrospective study of antibiotic resistance pattern of uropathogens especially Escherichia coli in north India. Cibtech journal of microbiology. 2014 vol. 3 (2) April-June, pp. 29-36.

3. Ana L.Flores-Mireles, Jennifer N.Walker, Michael Caparon and Scott J.Hultgren nary tract infections: Epidemiology, mechanisms of infection and treatment options. Nature Reviews Microbiology 13(5) - April 2015

4. Angus N. Oli et al. Bacteriology and Antibiogram of Urinary Tract Infection Among Female Patients in a Tertiary Health Facility in South Eastern Nigeria The Open Microbiology Journal, 2017, Volume 11293

5. Avinash Laghawe, Apoorva Tripathi and S B Saxena. Aetiology of Urinary Tract Infection and antimicrobial susceptibility pattern of urinary isolates in tertiary care hospital in Central India: A retrospective analysis. Int.J.Curr.Microbiol.App.Sci (2015) 4(4): 962-970.

6. Banerjee S and Padmashri VP (2011). The Study of Urinay Tract Infections and Antibiogram of Uropathogens in and around Ahmadnagar, Maharashtra. International Journal of Infectious Diseases 9(1)

7. Bauer AW, Kirby WM, Sherris JC and Turck M (1966). Antibiotic susceptibility testing by a standardized single disk method. American Journal of Clinical Pathololgy 45 493-6.

8. CLSI-Clinical and Laboratory Standards Institute 2016. Performance standards for antimicrobial susceptibility testing. Twenty-second informational supplement. Wayne, PA, USA.

9. Dash M, Padhi S, Mohanty I, Panda P and Parida B (2013). Antimicrobial resistance in pathogens causing urinary tract infections in a rural community of Odisha, India. Journal of Family \& Community Medicine 20 20-6.

10. Florian M. E. Wagenlehner, Udo Hoyme, Martin Kaase, Reinhard Fünfstück, Kurt G. Naber, Guido Schmiemann Dtsch Arztebl. Uncomplicated Urinary Tract Infections. Int 2011; 108(24): 415-23

11. Kothari A and Sagar V (2008). Antibiotic resistance in pathogens causing community acquired urinary tract infections in India: a multicenter study. Journal of Infection in Developing Countries 2(5) 354-358

12. Kumar Y, Sood S, Sharma A and Mani KR (2013). Antibiogram and characterization of resistance markers among Escherichia coli isolates from urinary tract infections. Journal of Infection in Developing Countries 7(7) 513-9.

13. LE Nicolle. Complicated urinary tract infection in adults. Can J Infect Dis. Med Microbiol 2005; 16(6 349-360.

14. Mohamoud d Dobabash et al. Antibiogram Sensitivity in Urinary Tract Infections (UTI) at El Batnan Medical Center- Tobruk - Libya. Urol Nephrol Open Access J 2017, 4(3): 1.

15. Monika Yadav et al Microbial spectrum of urinary tract infections and its antibiogram in a tertiary care hospital Int J Res Med Sci. 2017 Jun;5(6):2718-2722

16. Mulugeta Kibret, Bayeh Abera. Prevalence and antibiogram of bacterial isolates from urinary tract infections at Dessie Health Research Laboratory, Ethiopia Asian Pac J Trop Biomed 2014; 4(2): 164-168

17. Nasim Kashef, Gholamreza Esmaeeli Djavid, Sahba Shahbazi. Antimicrobial susceptibility patterns of community-acquired uropathogens in Tehran, Iran. J Infect Dev Ctries 2010; 2010; 4(4):202-206

18. Niranjan V, Malini A. Antimicrobial resistance pattern in Escherichia coli causing urinary tract infection among inpatients. Indian J Med Res. 2014;139(6):945.

19. R. Venkatesh Naik, Basavaraj V. Peerapur. A Retrospective Study of the Culture and Sensitivity Pattern of Urinary Tract Infection Causing Organisms at RIMS Teaching Hospital, Raichur, India. Int.J.Curr.Microbiol.App.Sci (2017) 6(10): 1530-1536.

20. Rajesh KR, Mathavi S, Priyadarsini RI. Prevalence of antimicrobial resistance in uropathogens and determining empirical therapy for urinary tract infections. International Journal. 2010 Oct;1(5):260.

21. Sood S and Gupta R (2012). Antibiotic Resistance Pattern of Community Acquired Uropathogens at a Tertiary Care Hospital in Jaipur, Rajasthan. Indian Journal of Community Medicine 37 39-44.

22. Swati Talari, Padmaja Udaykumar. A Retrospective Study to Assess the Resistance Profile Among Pathogens Causing Urinary Tract Infection In Pediatric Population At A Tertiary Hospital. Journal of Pharmacy and Biological Sciences: Volume 12, Issue 3 Ver. V (May - June 2017), PP 32-36. 